\section{Oscillatory Transpiration May Complicate Stomatal Conductance and Gas-exchange Measurements}

\author{
Mary Ann Rose'and Mark A. Rose ${ }^{2}$ \\ Department of Horticulture, The Pennsylvania State University, University \\ Park, PA 16802
}

Additional index words. Rosa hybrids, photosynthesis, sap-flow gauge, stomatal cycling

\begin{abstract}
A closed-loop photosynthesis system and a heat-balance sap-flow gauge independently confirmed oscillatory transpiration in a greenhouse-grown Rosa hybrids $\mathbf{L}$. Repetitive sampling revealed 60-minute synchronized oscillations in $\mathrm{CO}_{2}$-exchange rate, stomatal conductance, and whole-plant sap-flow rate. To avoid confusing cyclical plant responses with random noise in measurement, we suggest that gas-exchange protocols begin with frequent, repetitive measurements to determine whether transpiration is stable or oscillating. Single measurements of individual plants would be justified only when transpiration is steady state.
\end{abstract}

Oscillatory transpiration (OT) is a rhythmic fluctuation in transpiration that results from cyclic stomatal opening and closure; it has been described in many species (Barrs, 197 1). Although OT may be triggered by environmental stimuli (Hopmans, 1971), the rhythm is autonomic and does not mimic environmental rhythms (Ban-s, 1971). Research suggests that stomatal cycling maybe induced by internal fluctuations in water potential and resistance to flow within the plant (Barrs and Klepper, 1968; Hopmans, 1971). Barrs (1971) reported that for most plants that have been studied, the average period of the stomatal cycle is 10 to $50 \mathrm{~min}$.

Three independent methods (lysimeter, sapflow gauge, and infrared leaf-temperature sensor) confirmed the occurrence of OT in Rosa hybrids L. under high-intensity discharge (HID) lamps (Rose et al., 1994). Oscillatory transpiration was not an isolated phenomenon, having occurred some time during the shoot development cycle of all rose plants $(\mathrm{n}=$ 23) monitored to date (Rose, 1994). Although we have detected OT under natural daylight conditions, most of our experiments have been executed at night; the very stable irradiance, temperature, and humidity achieved in the nighttime greenhouse reduce the possibility that OT might be caused by an environmental rhythm. Further, commercial rose producers use HID lamps to increase productivity; supplemental irradiance maybe used all night long in the winter.

Our objective in this experiment was to demonstrate the importance of determining whether OT occurs while measuring gas exchange (GE). GE measurements are widely

Received for publication 14 July 1993. Accepted for publication 26 Dec. 1993. The cost of publishing this paper was defrayed in part by the payment of page charges. Under postal regulations, this paper therefore must be hereby marked advertisement solely to indicate this fact.

'Graduate Student.

${ }^{2}$ Senior Research Aide. used in horticultural research; HID lamps are often used during measurement to provide a constant source of irradiation.

A single potted 'Fire ' $\mathrm{N}$ Ice' rose was software (Dynamic Microsystems, Silver Spring, Md.). Microsystems, Silver Spring, Md.). selected from a group of nine flowering roses; all nine plants had exhibited OT (monitored by sap-flow gauges and lysimeters) during nightly irradiance periods at some time within the previous 30 days. Root-zone moisture tension did not exceed $6 \mathrm{kPa}$, and the pot was foilcovered to reduce evaporation. On 2 May 1993, GE measurement commenced at 2150 $\mathrm{HR}$, when the rose plant was irradiated with high-pressure sodium lamps. Stomatal conductance $\left(\mathrm{mol} \mathrm{CO} / \mathrm{m}^{2}\right.$ per see) and net $\mathrm{CO}_{2}$ exchange rate $\left(\mathrm{CER}, \mu \mathrm{mol} \mathrm{CO} / \mathrm{C}^{2}\right.$ per see) of the terminal leaflet of two fully mature leaves on separate branches were measured about every 10 min using a portable photosynthesis system (model 6200; LI-COR, Lincoln, Neb.); three consecutive 10-sec measurements were averaged. Environmental conditions were stable during measurements: average changes in leaf temperature and relative humidity $(\mathrm{RH})$ inside the 0.25-liter cuvette during each 10sec measurement were $<0.1 \mathrm{C}$ and $<0.6 \%$, respectively. During the experimental period (240 rein), the ranges in greenhouse air temperature and $\mathrm{RH}$ were 21.6 to $23.2 \mathrm{C}$ and $41.1 \%$ to $48.5 \%$, respectively. Oscillations in transpiration were confirmed independently of GE measurements by continuously monitoring

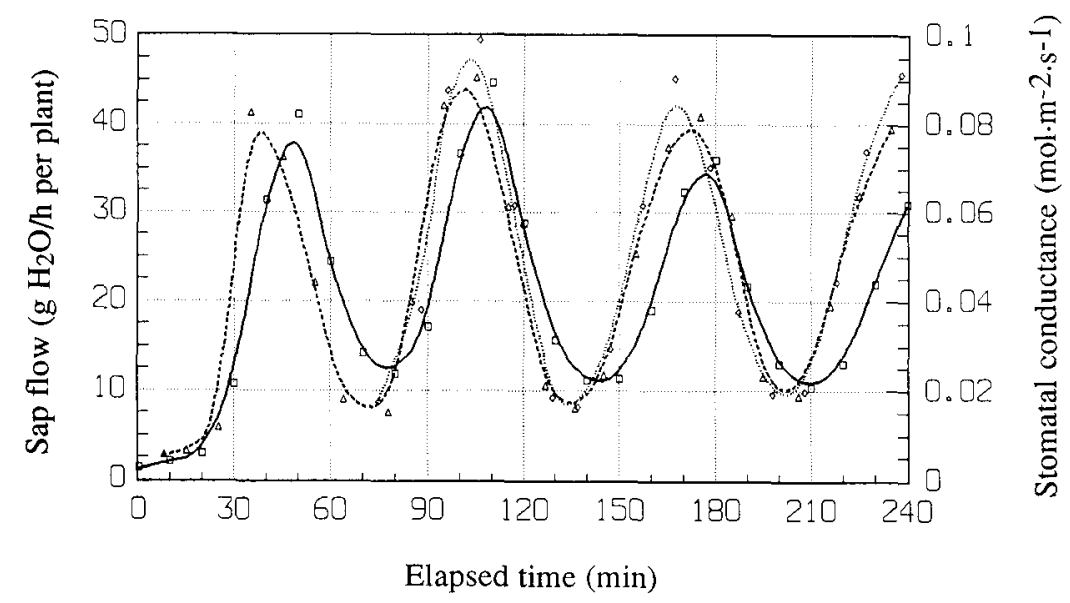

Fig. 1. Sap flow (-__— $)$ and stomatal conductances of leaf $1(---\Delta---)$ and leaf $2(\cdots \vee \cdots)$. High-pressure sodium lamps were turned on at time $=0 \mathrm{~min}$. The spline curves were fitted to the data using GB-STAT

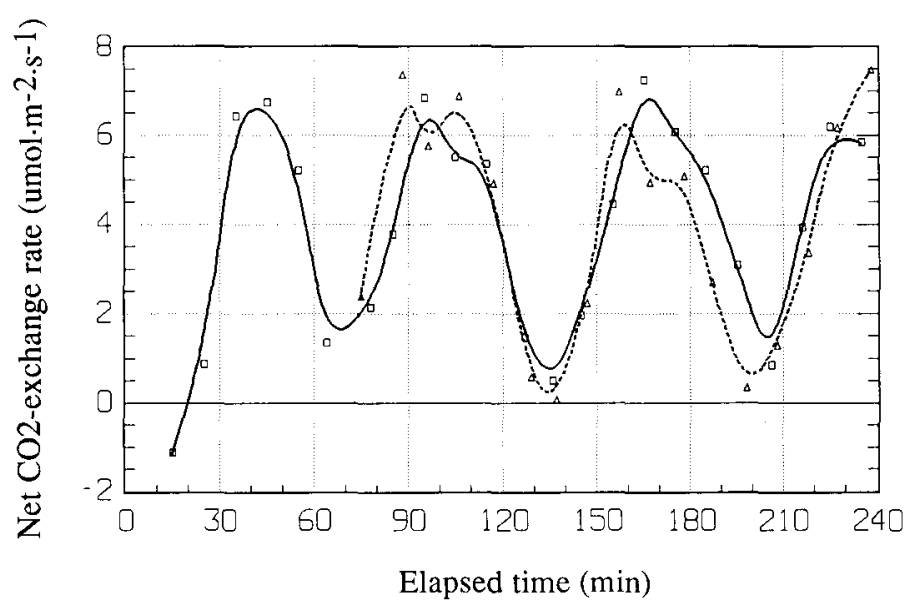

Fig. 2. Net $\mathrm{CO}_{2}$-exchange rates of leaf 1 ( $\square \square-$ - $)$ and leaf 2 (--- $\left.\Delta---\right)$. High-pressure sodium lamps were turned on at time $=0 \mathrm{~min}$. The spline cuves were fitted to the data using GB-STAT software (Dynamic 
whole-plant sap-flow rate $\left(\mathrm{g} \mathrm{H}_{2} \mathrm{O} / \mathrm{h}\right.$ per plant) with a heat-balance sap-flow gauge (model SGA10; Dynamax, Houston) mounted on the main stem. The rose had been pruned to a single main stem with multiple laterals.

The sap-flow rate in darkness during the hour before irradiation indicated a low basal rate of transpiration $\left(1.4 \pm 0.2 \mathrm{~g} \cdot \mathrm{h}^{-1}\right)$. Sustained oscillations in sap flow between 11 and 42 $\mathrm{g} \cdot \mathrm{h}^{-1}$ were induced by irradiation (Fig. 1). Stomatal conductance oscillated between 0.02 and $0.09 \mathrm{~mol} \cdot \mathrm{m}^{-2} \cdot \mathrm{s}^{-1}$; the waveform of the oscillation was similar to oscillations in sap flow in both phase and period (leaf 1, Fig. 1). Measurements of stomatal conductance in a second leaf (leaf 2, Fig. 1) were begun $75 \mathrm{~min}$ after lamps were turned on. The oscillatory patterns of stomatal conductance in leaf 1 and 2 were similar in phase, period, and amplitude. Ehrler et al. (1965) also observed that when OT occurred, the stomata] movements of leaves on a single plant were synchronized. The CER also oscillated in phase with stomata] conductance and had a similar $\approx 60-$ min period (Fig. 2). However, the waveform pattern in CER was irregular compared to conductance and sap flow (Fig. 2).

Even in controlled environments, the various control systems may cause rapid changes in environmental conditions that could trigger OT. Unless continuous measurement of GE is feasible, detecting whether OT occurs maybe critical for reliable estimates of average GE rates. For example, if our measurements had been taken at 60 -min intervals, commencing either 20 or $50 \mathrm{~min}$ after lamps were turned on, the measurements would have coincided with the minima or maxima of the sine-wave function (Fig.1); average conductance would have been $\approx 0.016+0.008$ or $\approx 0.075+0.011$ $\mathrm{mol} \cdot \mathrm{m}^{-2} \cdot \mathrm{s}^{-1}$, respectively. Further, an appropriate sampling period must be chosen to detect waveform phenomena; otherwise, the cyclical patterns in stomata] conductance, $\mathrm{CO}_{2}$ exchange, and sap flow might be obscured, and large deviations from the mean that could result might be interpreted as instrumental "noise."

Averaging all 10-min data, average stomatal conductance and sap-flow rate of the oscillating plant were $0.045 \pm 0.029 \mathrm{~mol} \cdot \mathrm{m}^{-2} \cdot \mathrm{s}^{-1}$ and $19.4 \pm 12.3 \mathrm{~g} \cdot \mathrm{h}^{-1}$, respectively. An adjacent rose plant was measured concurrently at 10min intervals for stomata] conductance and sap-flow rate (data not shown). These measurements indicated that OT was not occurring in this plant; however, its average stomatal conductance and sap-flow rate were close to those of the plant exhibiting OT $(0.039 \pm 0.003$ $\mathrm{mol} \cdot \mathrm{m}^{-2} \cdot \mathrm{s}^{-1}$ and $\left.18.2 \pm 2.0 \mathrm{~g} \cdot \mathrm{h}^{-1}\right)$. The much smaller standard deviations for the nonoscillating plant are evidence of its steady-state transpiration rate. The occurrence and absence of cyclical behavior in adjacent plants demonstrated the unpredictability of OT.

In many scientific studies, average GE rate for a group of plants has been determined by taking one measurement per plant. While one measurement per plant may suffice when transpiration rates are stable, our study demonstrates the weakness in that approach if OT occurs in some or all plants. We suggest that
GE protocols begin by determining whether transpiration is stable or oscillating; this may require frequent, repetitive measurements on a sample population of plants (as large as practical). To characterize an oscillation, intervals between GE measurements must be shorter than the period of oscillation. Investigators should also consider whether repeatedly enclosing" a leaf in the GE cuvette may cause stomatal closure. Based on sap-flow monitoring, the stomatal apparatus of rose did not appear to be disturbed by the $\approx 30$-sec enclosures taken at 10-min intervals. Thus, we were able to monitor oscillations accurately.

\section{Literature Cited}

Barrs, H.D, 1971. Cyclic variations in stomatal aperture,transpiration, and leaf water potential under constant environmental conditions. Annu. Rev. Plant Physiol. 22:223-236.

Barrs,H.D.and B. Klepper. 1968. Cyclic variations inplant properties under constant environmentalconditions.Physiol. Plant. 21:711-730.

Ehrler, W.L., F.S. Nakayama, and C.H. van Bavel. 1965.Cyclicchangesin water balance and transpiration of cotton leaves in a steady environment. Physiol. Plant. 18:766-775.

Hopmans,P.A.1971. Rhythms in stomatal opening of bean leaves. Meded. Landbouwhogesch Wageningen 71 , no. 3:1-86.

Rose, Mark A. 1994. Monitoring rose oscillatory transpiration by integrating a greenhouse climatecomputer and physiological sensors, $\mathrm{PhD}$ Diss., Pennsylvania State Univ., University Park.

Rose, Mark A., D.J. Beattie, and J.W. White, 1994. Oscillations of whole-plant transpiration in 'Moonlight' rose, J. Amer. Soc. Hort. Sci. 119:439-445. 